\title{
A national survey of attitudes to COVID-19 digital contact tracing in the Republic of Ireland
}

\author{
Michael Edmund O'Callaghan ${ }^{1}$ (1) Jim Buckley ${ }^{2,3} \cdot$ Brian Fitzgerald $^{2} \cdot$ Kevin Johnson $^{4} \cdot$ John Laffey $^{5}$. \\ Bairbre McNicholas ${ }^{5}$ - Bashar Nuseibeh ${ }^{2}$ - Derek O'Keeffe ${ }^{5} \cdot$ lan O'Keeffe $^{2}$ - Abdul Razzaq ${ }^{2} \cdot$ Kaavya Rekanar $^{2}$. \\ Ita Richardson ${ }^{2} \cdot$ Andrew Simpkin $^{6} \cdot$ Jaynal Abedin $^{6} \cdot$ Cristiano Storni $^{3} \cdot$ Damyanka Tsvyatkova $^{2} \cdot$ Jane Walsh $^{7}$. \\ Thomas Welsh ${ }^{2} \cdot$ Liam Glynn ${ }^{1,8}$
}

Received: 2 July 2020 / Accepted: 23 September 2020 / Published online: 16 October 2020

(C) Royal Academy of Medicine in Ireland 2020

\begin{abstract}
Background Contact tracing remains a critical part of controlling COVID-19 spread. Many countries have developed novel software applications (Apps) in an effort to augment traditional contact tracing methods.

Aim Conduct a national survey of the Irish population to examine barriers and levers to the use of a contact tracing App.

Methods Adult participants were invited to respond via an online survey weblink sent via e-mail and messaging Apps and posted on our university website and on popular social media platforms, prior to launch of the national App solution.

Results A total of 8088 responses were received, with all 26 counties of the Republic of Ireland represented. Fifty-four percent of respondents said they would definitely download a contact-tracing App, while 30\% said they would probably download a contact tracing App. Ninety-five percent of respondents identified at least one reason for them to download such an App, with the most common reasons being the potential for the App to help family members and friends and a sense of responsibility to the wider community. Fifty-nine percent identified at least one reason not to download the App, with the most common reasons being fear that technology companies or the government might use the App technology for greater surveillance after the pandemic.

Conclusion The Irish citizens surveyed expressed high levels of willingness to download a public health-backed App to augment contact tracing. Concerns raised regarding privacy and data security will be critical if the App is to achieve the large-scale adoption and ongoing use required for its effective operation.
\end{abstract}

Keywords App $\cdot$ Contact tracing $\cdot$ Coronavirus $\cdot$ COVID-19 $\cdot$ Online survey $\cdot$ Public opinion

Michael Edmund O'Callaghan

mike.ocallaghan@ul.ie

School of Medicine, University of Limerick, Limerick, Ireland

2 Lero - The Irish Software Research Centre, Tierney Building, University of Limerick, Limerick, Ireland

3 Department of Computer Science and Information Systems, University of Limerick, Limerick, Ireland

4 College of Education \& Health Sciences, University of Limerick, Limerick, Ireland

5 School of Medicine, National University of Ireland Galway (NUIG), Galway, Ireland

6 School of Mathematics, Statistics and Applied Mathematics, National University of Ireland Galway, Galway, Ireland

7 School of Psychology, National University of Ireland Galway, Galway, Ireland

8 Health Research Institute, University of Limerick, Limerick, Ireland

\section{Introduction}

Until a vaccine or efficacious treatment emerge, the key to limiting the harm caused by severe acute respiratory syndrome coronavirus 2 (SARS-CoV-2) and its associated disease (COVID19 ) is to identify and quarantine infected individuals as quickly as possible. China and neighboring countries have shown that an aggressive public health response involving rapid isolation of infected people and their close contacts can help contain the virus, thus reducing the number of patients requiring hospitallevel care at any one time $[1,2]$. Asymptomatic and presymptomatic spread of COVID-19 further underlines the importance of timely and effective contact tracing [3, 4].

Given the scale and gravity of the problem, novel software applications ("Apps") that can potentially simplify the laborious work of contact tracing are a tempting prospect [5]. These Apps use modern smartphone technology to record details of 
other smartphones in the user's vicinity, record smartphone location, or both, to determine the recent contacts of those who subsequently test positive for the virus [6]. While the evidence for contact tracing Apps is limited, in a global pandemic with a novel virus, many countries have designed and deployed Apps before their efficacy can be proven [7].

Researchers from the University of Oxford have estimated that if $56 \%$ of people were to download an ideal contacttracing App in the UK, this would be enough to control the disease by itself [8]. However, the authors clearly state in the same document, "lower numbers of App users will also have a positive effect." Indeed, the designers of Singapore's TraceTogether App state that these Apps should be viewed as an adjunct to a human-fronted process and not a panacea to end the pandemic [9].

The technological and privacy challenges associated with such Apps are considerable, and there is much for governments and public health bodies to consider [10,11]. The announcement of the "Exposure Notification" Application Programming Interface (API) as a joint venture by software companies Google and Apple on 20 May 2020 introduces a more direct means to access the Bluetooth technology harnessed by earlier Apps $[12,13]$. The Irish government have committed to building a free-to-download, free-to-use Irish contact-tracing App based on the Exposure Notification API. The initial specification document states that anyone will be able to use the App if they have a smartphone that is "less than 5 years old" [14].

It remains unclear what proportion of a population must use a contact tracing App for it to make an effective contribution to a COVID-19 response. It will be dependent on many factors, including the App's technological efficacy and the burden of COVID-19 in communities. However, what is clear is that any contact tracing App solution can only be effective if it is trusted, downloaded, and used by a significant number of citizens. The more people that download and use the App, the more the number of contacts between people will be silently logged by the App.

This study aims to gather "a priori" evidence from the Irish general public via an online survey concerning contact tracing Apps and relevant barriers deemed important to the adoption of such technologies, before any such App was available. While a pandemic of this scale has not been witnessed for more than a century, this is the third worrying outbreak of a potentially lethal coronavirus in the past 20 years [15]. How long countries will have to fight to suppress COVID-19 is unknown, yet it is likely that lessons learned from this pandemic, including those around use of technology, will prove useful for future infectious disease outbreaks.

\section{Objective}

The aim of this research is to conduct a national online survey with a large sample of the Irish population to examine barriers and levers to the use of a contact-tracing App to aid in the suppression of the COVID-19 pandemic.

\section{Methods}

A 37-item online survey was designed and piloted by researchers at the University of Limerick Graduate Entry Medical School (GEMS), Science Foundation Ireland Research Centre for Software (LERO), and the National University of Ireland Galway (NUIG) College of Medicine, Nursing and Health Sciences.

The final survey (see Appendix) was released via Qualtrics XM survey software [16] and featured five questions adapted from the Imperial College London "Public Response to UK Government Recommendations on COVID-19" population survey [17] and four questions adapted from the Oxford University survey of acceptability of a contact-tracing App [18].

The survey was released through social media platforms (Twitter, Facebook, LinkedIN, and Instagram), the University of Limerick website, and university e-mailing lists and via WhatsApp Messenger group conversations on Friday, 22 May, at $12 \mathrm{p} . \mathrm{m}$. The survey was live for 7 days, and the respondents were advised they needed to be aged 18 or older when taking the survey. While plans to develop an Irish contact-tracing App had been released in April [14], the Irish health service-endorsed contact-tracing App solution was launched in early July.

In light of the potential for self-selection bias of respondents more familiar with technology, respondents were encouraged to discuss the survey with friends and family so that the survey could be completed on behalf of another person who may not be regularly online. The survey was carried out during the first week of easing of widespread societal restrictions which had been in place in the RoI for the previous 10 weeks.

Ethical approval for this project was granted by the University of Limerick Education and Health Sciences (EHS) ethics committee [study ID 2020_04_18_EHS (ER), approved 21 May 2020].

\section{Statistical analysis}

All analyses were carried out in R v3.6 [19]. Descriptive statistics are used to present the findings of demographics and attitudes of the respondents to the COVID-19 crisis, contact tracing, and the relevant technologies.

For the primary measure of interest, willingness to download the App, the survey data were weighted by gender and education to better reflect the Irish population. The population distribution of gender (50\% male and female) and education ( $42 \%$ tertiary, $45 \%$ secondary, $13 \%$ primary or no formal education) was taken from census data recorded by the Central Statistics Office of Ireland [20]. 
We report univariate summaries of key survey questions, with the primary response being willingness to install the App. Chi-squared tests for association were carried out to investigate whether gender, age group, education level, or level of worry regarding COVID-19 were related to willingness to install.

\section{Results}

A total of 8088 complete responses were recorded. Just 27 responses were returned on behalf of others. Responses were received from all 26 counties in the Republic of Ireland, with most responses received from the major population centers of Dublin, Cork, Limerick, and Galway. The median age of respondents was 39 years, and mean age was 41 years. Eightyone percent of respondents were female. Regarding highest educational attainment, 7124 (87\%) of respondents reported having a 3 rd level qualification.

Overall physical and mental health was reported to be "very good" by 4249 (53\%) respondents, while 3208 (40\%) felt their health was "moderately good." Regarding chronic disease, 2519 (31\%) of respondents indicated they had at least one chronic health issue. SARS-CoV-2-related anxiety in the previous week was assessed, with $878(11 \%)$ of respondents reporting they had been "very worried," while 4588 (57\%) were "moderately worried."

When asked about contact tracing, 7929 (98\%) respondents understood the concept, while 7770 (96\%) agreed they would tell public health doctors where they had been and who they had met recently, if it was important to stop the spread of COVID-19.

Just 6 respondents indicated they do not own a mobile phone. Of the mobile phone owners, 8036 (98\%) own a smartphone, $7863(98 \%)$ of these respondents had downloaded an App before, and 7781 (97\%) stated that they would not need help to download an App to their smartphone. Just over two-thirds (68\%) of smartphone owners indicated they keep their phones "very close by (e.g., in pocket, bag or on the desk) all the time". A further 2211 (28\%) indicated they keep their smartphones "very close by (e.g., in pocket, bag or on the desk) more than $50 \%$ of the time.".

The majority of respondents ( 7757 or $96 \%$ ) indicated they understand what Bluetooth technology is used for, and 7921 (98\%) know how to turn on this function on their phone. Fiftyfive percent of respondents (4444) stated that they believed Bluetooth adversely affects device battery life. Bluetooth use was common among owners of smartphones, with 4995 (63\%) using it "Everyday" or "Most days."

Use of geolocation technology was reported by 7026 $(89 \%)$ of smartphone users. Of smartphone users, 5931 (75\%) would be willing to allow the App to access the geolocation capabilities of their phone.
Respondents were asked to select from a list of options reasons that would encourage them to install the App (see Table 1). In rank order, 6395 (79\%) felt the App would "help protect my family and friends", 6338 (78\%) felt "a sense of responsibility to the wider community," while 5725 (71\%) felt it would "let me know my risk of being infected." Additional free text responses included "if the App was shown to be effective" and "if it was shown to be secure."

While 3335 (41\%) of respondents could see no reason not to install the App, the remaining 59\% of respondents selected at least one option from a list of 10 options. "I worry technology companies will use this as an excuse for greater surveillance after the pandemic" was selected by 3342 (41\%) respondents, "I worry the government would use this as an excuse for greater surveillance after the pandemic" was selected by 2636 (33\%) people, and "I worry that my phone would be more likely to get hacked" was selected by $1742(22 \%)$ of respondents.

In terms of preference for choice of the App technology, 3907 (48\%) of total respondents stated it did not matter to them. For the 4181 who expressed a preference, 1569 (38\% of this group) preferred a Bluetooth-only App, 1344 (32\%) were unsure, $738(18 \%)$ would like an App that uses Bluetooth and Geolocation technology, and 530 (13\%) would like to see a Geolocation-only App.

\section{Weighted analyses}

Initially, 58\% percent of respondents indicated they "definitely will install" the App, with a further $25 \%$ indicating they "probably will install" the App. Eight percent indicated they "may or may not install", $3 \%$ indicated they "probably won't install," while $6 \%$ stated they "definitely won't install" the App. Following several questions containing more detailed information about the technologies involved in contacttracing Apps and some potential push and pull factors regarding adoption (see Table 1), 54\% of respondents indicated they definitely will install the App, 30\% indicated they probably will install the App, and 7\% indicated they may or may not install the App. Selection of the two remaining options for this question did not change.

There is evidence for an association between gender and unwillingness to install the App, with males more likely to respond that they probably or definitely will not install the app (11\% vs $6 \%$ in females). There is also evidence for an association with age group, with the oldest and youngest groups most likely to indicate they probably or definitely will install the App. Finally, COVID-related worry was associated with willingness to install - those "Not At All Worried" about COVID were far more likely to respond that they definitely will not install the App (34\% vs $6 \%$ or lower in those more worried about COVID). 
Table 1 Barriers/levers encouraging and discouraging App download

Total responses

$\%$ respondents

Reasons that would make it more likely for you to install the App (you can select many answers)

It would help protect my family and friends

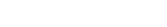

A sense of responsibility to the wider community

6338

78.4

It would let me know my risk of being infected

5725

It would help protect me

5239

5148

It would help reduce the number of deaths among older people

4496

It might stop the epidemic

437

None of these apply to me

$305 \quad 3.8$

64.8

Other (please specify)

Reasons that would make it less likely for you to install the App (you can select many answer)

I worry the technology companies would use this as an excuse for greater surveillance after the pandemic

3342

3335

2636

I worry the government would use this as an excuse for greater surveillance after the pandemic

I worry that my phone would be more likely to get hacked

1742

653

645

I would not give the HSE access to information about which locations I have visited recently

I would not give the HSE access to information about which phones or people I have been near recently

609

350

Other (please specify)

I would not benefit from the App

I've already had COVID-19

112

102

The App would be too much hassle to install

99

I will not be infected anyway

\section{Discussion}

\section{Summary of main findings}

This survey has found considerable willingness among Irish citizens to download a public health service-endorsed COVID-19 contact tracing App. It was an acceptable intervention to a large majority $(82 \%)$ of our sample. This did not alter significantly following mention of the type of technology involved or issues surrounding tracing of close contacts or location data.

Our sample was heavily skewed toward female participants; those with higher educational attainment and the 18-29-year and 30-41-year age groups were overrepresented when compared with the Irish population. This is partly explained by our survey invitation gaining traction with the online "followers" of a popular female Irish general practitioner who encouraged completion of the survey. This single endorsement was followed by almost 3000 responses being received from young females in a 24-h period. However, weighting for gender did not significantly change the primary outcome measure of willingness to download the App. Forty-two percent of Irish citizens possess a third-level qualification, compared with $82 \%$ of our cohort. Higher educational attainment has an age gradient in Ireland [20], and thus, this may be partly explained by the fact our sample was younger than the population, yet likely also reflects our chosen methodology where participants were primarily recruited using online platforms.

Ninety-two percent of our cohort reported good or very good health, which is greater than the $84 \%$ seen in the
Healthy Ireland survey report 2019 [21]. Regarding chronic disease, $69 \%$ of our cohort reported no chronic illness, which is in line with the $68 \%$ reported in the Health Ireland survey. Poorer self-reported health status did not translate to increased willingness to download a contact-tracing App. Indeed, those with very good health were most likely to "definitely" download the App. However, respondents' level of worry about COVID-19 in the past week did influence expressed willingness to download the App. While $2 \%$ of those very worried about COVID-19 would definitely not install the App, 38\% of the 478 respondents not at all worried about COVID-19 said they definitely would not install the App. This may be a relevant area for public awareness campaigns when the Irish App is released.

Smartphone ownership of $98 \%$ is higher than the $91 \%$ reported in the 2019 Global Mobile Consumer Survey [22] and may be explained by the ease with which online surveys can be taken using a smartphone's Internet browser. That $96 \%$ of respondents keep their phone very close by more than $50 \%$ of the time is important if detection of a smartphone's Bluetooth signal by the App will act a proxy for its owner's precise location.

While a number of responses were received from people aged 65 and above $(563(7 \%))$, very few responses were received on behalf of other people. It was hoped to capture more opinions from less technologically adept citizens in this survey by encouraging respondents to discuss it with friends and family. Of the people aged 65 and above, $14 \%$ were very worried about COVID-19, 91\% had a smartphone, and 91\% said they would probably or definitely install the contact-tracing App. 
While selection bias and other sampling issues inherent in online surveys are well described, online survey methods are quick and can be used to reach a wide audience [23, 24]. The latter characteristics of online surveys were attractive in the early stages of this pandemic and have allowed us to quickly inform a national discussion concerning the Irish contacttracing App. However, planned qualitative work will be critical to gather opinions and attitudes of underrepresented and hard-to-reach cohorts if App deployment is to avoid a digital divide that mirrors well-established socioeconomic patterns of engagement with the healthcare system.

A good level of knowledge of Bluetooth technology and prior use of Bluetooth technology was reported by the majority of our respondents. However, if the Google/Apple Exposure Notification API bypasses the manual step of turning on Bluetooth and enabling the App to access it, these findings may not be as relevant. A large portion (3907 $(48 \%)$ ) of respondents indicated they had no preference for the technology the Irish contact-tracing App uses. However, an App featuring geolocation technology was only preferred by $16 \%$ of respondents. One quarter of respondents indicated they would not permit a contact-tracing App to access geolocation data. While fully anonymized geolocation data in the context of the COVID-19 pandemic is not likely to cause any data protection issues per se [25], it may hinder adoption of the App among some cohorts of the population, particularly given the types of barriers described in Table 1 .

\section{Strengths and limitations}

This study captured data from a large sample of the general public in the Republic of Ireland using an online survey tool. However, our sample comprised a higher proportion of smartphone owners than is seen nationally, and selection bias effects regarding more technologically adept citizens are a limitation. If those taking part in this study are more or less likely to download the App compared with those who did not take part, then willingness to download at a population level may be different to this study's estimates. Given the nature of the online survey, our results may represent an upper bound for willingness to install the app. Additionally, our sample was younger than the Irish population, comprised more females, and had a higher level of educational attainment than the Irish population. However, given the size of our sample, weighting to resolve these sampling issues was possible and did not significantly alter our main findings (see Table 2).

The survey was deployed at a time of considerable uncertainty where the optimal response to the pandemic and the role that technology might play was unclear $[10,11]$. Expressions of concern around potential privacy issues associated with contacttracing Apps [26, 27] and discussion around potential, but as yet unproven, benefits [28] were reported widely in the mainstream media around this time, which may have influenced results.

\section{Implications for research and practice}

It seems that the primary driver for people's willingness to download a public health-backed contact-tracing App during the current crisis is a desire to help others and "for the greater good." Forty-one percent of our sample did not feel any of our presented options would dissuade them from downloading the App (see Table 1). However, privacy issues and worries regarding technology companies, governments, and hackers capitalizing on perceived security weaknesses resulting from such an App were expressed by many respondents. Plans to release the source code of the Irish contact-tracing App are welcome and may help reassure people in this regard [14].

Analysis of free text responses yielded interesting insights and suggestions for the App. Some respondents stated that they would be more likely to install the App if there was clear evidence that it was effective. There were also several technology-based suggestions for the App. Battery life concerns led some respondents to suggest integrating a feature which automatically enables Bluetooth when the user leaves their primary residence or workplace (e.g., the App is activated when the phone is disconnected from home or work WiFi). Another suggestion involves pre-setting times for the App to be active, which correspond with their work, travel, or shopping schedule in an effort to conserve battery life. Finally, use of Bluetooth Low Energy (BLE) technology where possible may be another worthwhile feature.

Assessing willingness to download contact tracing Apps has been estimated by large surveys in the UK, Italy, Germany, France, and the USA. In keeping with our study, $75 \%$ of respondents in these countries indicated comparable willingness to download a similar contact tracing App [29]. How intent to download translates to actual downloads and ongoing use remains to be seen as contact-tracing Apps in the countries listed above are all in development or in early stages of deployment.

However, Singapore deployed their TraceTogether App in late March and 10 weeks later, as of early June, $28 \%$ of Singaporeans have downloaded the App [30]. In Australia, $64 \%$ of respondents in a large survey conducted in early April stated they would download a Bluetooth tracking App [31]. Follow up survey by the same research group 12 days after launch of the Australia "COVIDSafe" App established that $44 \%$ of respondents had downloaded the App [32]. Of respondents who downloaded the App, 1 in 10 was not using it. At a population level, it is estimated that approximately six million Australians (20\% of the population) have downloaded the COVIDSafe App [33]. Iceland is considered to have had the most successful adoption of a national contact-tracing App, and currently, almost $40 \%$ of their population of 340,000 has voluntarily downloaded the Rakning-19 App 


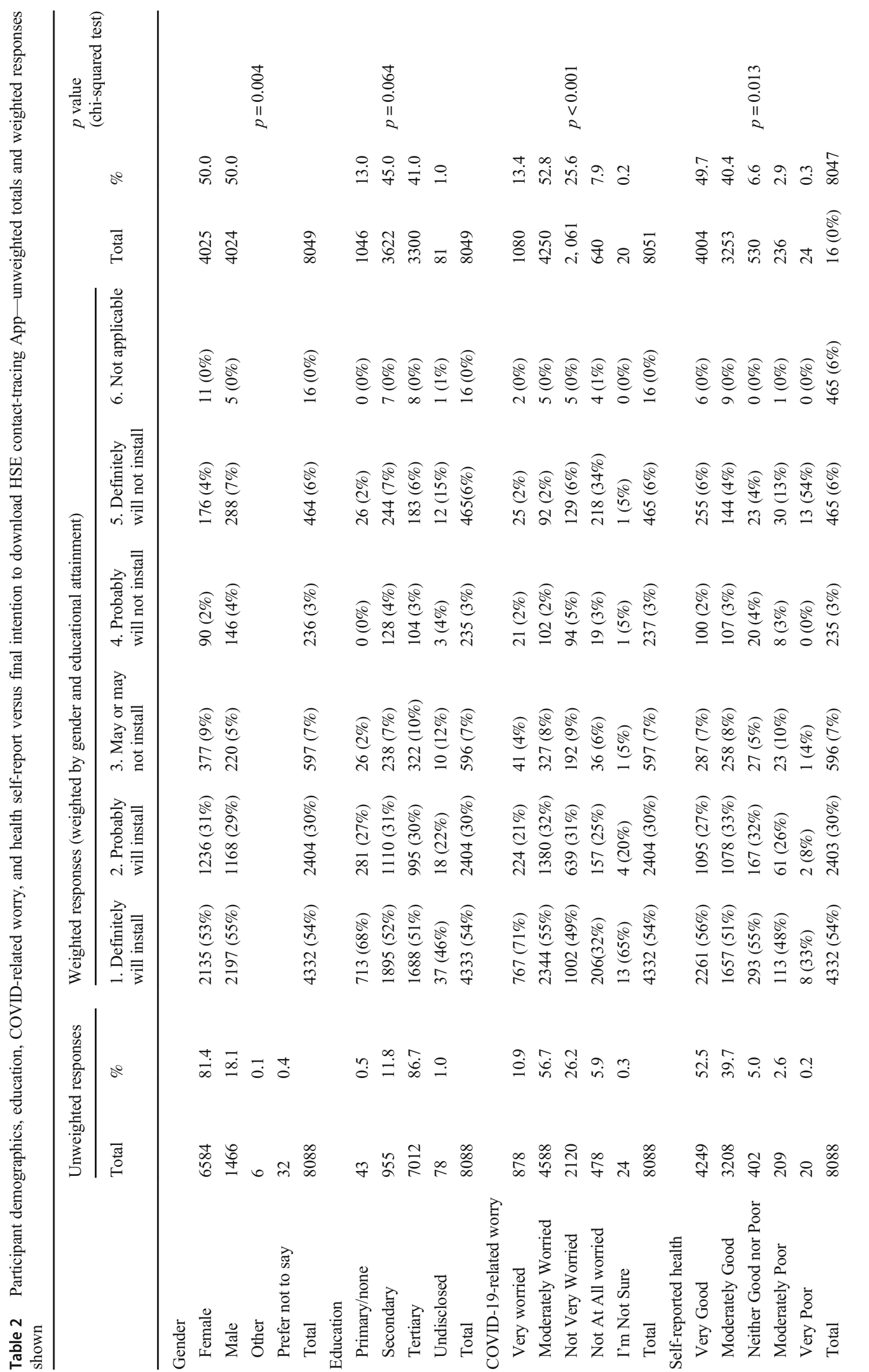


$[34,35]$. China and India have made use of COVID-19 Apps mandatory [36], but it is unlikely western democracies will opt for this strategy.

Each country's response thus far to COVID-19 is so varied, from testing to media reporting, that it is no surprise that a onesize-fits-all approach is unlikely to exist for COVID-19 contact tracing Apps. There are a number of technological issues that are likely to negatively impact on download and usage rates. The App being developed for the Republic of Ireland will only work on newer smartphones, and all smartphone users will need to update their operating system to install the new API being developed by Google/Apple. Each of the barriers described here presents an additional hurdle for an undetermined proportion of the population, and each will lead to incremental reductions in App usage.

\section{Conclusion}

To date, the international evidence for contact tracing Apps remains limited. Despite this, our study indicates a significant majority of the Irish general public express a willingness to download an App which aims to augment our existing contact tracing process. Issues raised around privacy, data protection, and some mistrust of technology companies and government remind us that any App will need to have transparency at its core and defined timelines for operation.

App download and ongoing use are two separate challenges, and to ensure both occur, it will be important to generate evidence that this App is useful to our country's contact tracing efforts. While it is difficult to quantify the benefit of a single measure when several measures are simultaneously active, it may be beneficial to keep the public informed on key data relating to the App, including downloads, active users, and numbers of cases where the App has helped contact tracing efforts as a method of promoting adoption and ongoing use. People have indicated a clear willingness to help, but experience from other countries shows that such willingness does not always translate into action. Allowing the general public to see in real time the public health benefits of this App may help promote the App and maintain public interest.

Irish citizens surveyed are very familiar with Bluetooth technology and expressed a preference for Bluetooth-based App rather than a geolocation-based App. Given this, and the additional data protection concerns associated with geolocation data [37], the plans for the Irish App to use Bluetooth technology are likely to be more acceptable to the Irish public. However, messaging around privacy, data protection, and the role that the Google/Apple API plays in the App design will be important. Our results also suggest public health campaigns encouraging use of the App should focus on the key messages of the seriousness of COVID-19 disease and around the benefit to family, friends, and communities of reducing COVID-19 spread. How effective public health messaging will be in encouraging download and ongoing use of the App as the pandemic progresses remains to be seen.

Data from other countries suggest a significant response to contact tracing Apps by early adopters, followed by a swift plateauing in the population. In addition, as countries continue to reduce transmission and overall healthcare burden from COVID-19 effectively, general societal concern is likely to decline. However, with the considerable uncertainty that prevails with the COVID-19 pandemic and the likelihood of further outbreaks, it seems prudent for all countries to continue contact tracing App development and refinement.

The authors of this study will repeat this survey in September 2020, approximately 10 weeks after the Irish contact-tracing App has been launched.

Acknowledgments We thank Prof. Helen Ward and Dr. Christina Atchison and the research team in the Patient Experience Research Centre (PERC) of Imperial College London, School of Public Health, for the permission to use 5 questions from their survey instrument [17]. Imperial College London, in turn, thanks Prof. Samuel Yeung Shan Wong, Prof. Kin On Kwok, and Ms. Wan In Wei from JC School of Public Health and Primary Care, The Chinese University of Hong Kong, Hong Kong Special Administrative Region, China, for the permission to use their survey instrument and translating it into English (Kwok et al.).

Authors' contributions MOC, LG, and JB conceived and designed this study, interpreted the data, drafted the manuscript, and revised the manuscript for important intellectual content. MOC designed and deployed the final survey instrument. AS interpreted the data and provided statistical testing expertise and advice and revised the manuscript for important intellectual content. DOK, JW, BN, and CS aided in the interpretation of the data and revised the manuscript for important intellectual content. All of the authors reviewed, discussed, and approved the final manuscript.

Funding This research work is supported by a funding grant from Science Foundation Ireland (13/RC/2094) and the COVIGILANT Science Foundation Ireland grant Project ID: 20/COV/0133.

\section{Compliance with ethical standards}

Ethical approval Ethical approval for this project was granted by the University of Limerick Education and Health Sciences (EHS) ethics committee [study ID 2020_04_18_EHS (ER)].

The University of Limerick EHS ethics committee is "charged by the University to consider the ethics of proposed research projects which will involve human subjects and to agree or not, as is the case, as to whether the projected research is ethical." Approval was granted for our project on 21st May 2020.

Statement on participant consent Participants were asked to tick a box on the online survey following the Survey Introduction (see first page of Appendix 1) to indicate their consent to participate in our research study. All responses were anonymous.

Conflict of interest The authors declare that they have no conflicts of interest. 


\section{Appendix 1}

\section{COVID-19 Online Survey}

Thank you for taking the time to check out our survey on COVID-19. We're keen to hear if you think technology has a role in the Irish response to this pandemic...

Dr Michael O'Callaghan GP + Researcher

Prof Liam Glynn GP + Professor of General Practice University of Limerick, Ireland

Before you take the survey, please read the following:

Study Title: "COVID-19 response in Ireland: is there a role for technology?" Principal Investigator: Dr Michael O'Callaghan (University of Limerick) Background information: The SARS-CoV-2 Coronavirus (which causes the disease COVID-19) was declared a global health emergency by the World Health Organisation on 11th March 2020. An important part of limiting transmission of SARS-CoV-2 involves "contact tracing", where people who catch the virus are asked who they have been in contact with recently. This is particularly important for COVID-19 as many people who are infected will have no symptoms. What is the reason for this study?: Other countries are already using software applications ("Apps") which people can download to their smartphones to help with contact tracing efforts. Some of these Apps will remember the places where you have been, while others only remember other smartphones that you have come into close contact with in the past 14 days. This study aims to understand more about the experience of the Irish general public of this pandemic so far, including how it has changed how we use technology. We also wish to find out if people feel a smartphone App might be an appropriate way to help our public health teams with their contact tracing efforts. Why am I being asked to take part?: In order for these Apps to be effective a large number of Irish people would need to download the App and allow it to store information about which smartphones they have been close to in the past 14 days. By taking this survey you will help us figure out what the general public in Ireland think of a Contact Tracing App. What will you have to do?: We are asking you complete an anonymous survey. Answering these questions should take approximately 10-15 minutes of your time. If you do decide to take the survey, you may withdraw at any time for any reason by just closing your Internet browser. What are the benefits?: Your answers will help us understand more about how COVID-19 is affecting people in Ireland and what people think about using technology to help us in our response to this virus. What are the risks?: You might decide that you don't want to answer a question. If this happens, most questions feature a "I'm not sure" option that will skip to the next question. What happens to the information?: Your answers will be completely anonymous. These answers will be stored in a password-protected file on a University of Limerick password-protected computer for the duration of this study. The answers we collect may be used in academic publications and in online reports as we try to summarise participants' opinions on the questions we are asking in this survey. What if you have more questions?: If you would like to ask us any questions before deciding to take part, please contact the research team using the email address mike.ocallaghan@ul.ie Participant Consent Should you agree to participate in this study please read the statements below and if you agree to them, please tick the box below: - I have read and understood the participant information above. $\bullet$ I confirm that I am aged 18 years or over and live on the island of Ireland. $\bullet$ I am fully aware of what I will have to do, and of any risks and benefits of the study. • I understand what the project is 
about, and what the results will be used for. - I understand that what the researchers find out in this study may be shared with others but that all answers I give will be anonymous. $\bullet$ I know that I am choosing to take part in the study and that I can stop taking part in the study at any stage without giving any reason by closing this browser window.

I agree to the statements above and I consent to taking part in this research study (1)

Q1 Are you taking this survey for yourself or on behalf of someone else?

Myself (1)

Someone else (2)

You have indicated you are answering the survey questions on behalf of someone else. Please answer ALL the following questions from THEIR point of view...

Q2 What is your age?

Q3 What is your Gender? (please select option that best applies)

Male (1)

Female (2)

Other (3)

Prefer not to say (4) 
Q4 What is your highest level of educational attainment? (if a student, please indicate what qualification you hope to achieve)

No formal education or training (1)

Finished primary school (4)

Finished secondary school (5)

Skilled trade (6)

3rd level qualification (7)

Prefer not to say (8)

Q5 In which county do you live?

$\boldsymbol{\nabla}$ Antrim (1) ... Not applicable (34)

Q6 In general, how good or how poor would you say your current state of health is (i.e. mental and physical)?

Very good (1)

Moderately good (2)

Neither good nor poor (3)

Moderately poor (4)

Very poor (5)

Q7 Which, if any, of the following chronic (or ongoing) health conditions do you currently have? Please select all that apply. If you do not currently have a chronic or ongoing health condition, please select 
the 'Not applicable' option. For the following questions, please remember that your answers are always treated confidentially and are never analysed individually. However, we have provided you with a "Prefer not to say" option if you would rather not share your experiences.

QNot Applicable, no ongoing health issue (1)

Heart Condition (2)

Lung Condition (3)

Diabetes (4)

Neurological Condition (5)

Gut/Tummy/Bowel Condition (6)

Currently undergoing cancer treatment (7)

Mental health condition (8)

Other (please specify below) (9)

Prefer not to say (10)

Q8 Does anyone else in your household, or who you have caring responsibilities for, have any chronic / ongoing health conditions?

Yes (1)

No (2)

Not Applicable (3)

Prefer not to say (4) 
Q9 The 2019 coronavirus, otherwise known as COVID-19, is an infectious disease first identified in the city of Wuhan, China. Infections have since been reported around the world. Symptoms include fever, coughing and breathing difficulties. In the past week, how worried have you been about the current coronavirus (i.e. COVID-19) outbreak in Ireland?

Very Worried (1)

Moderately Worried (2)

Not Very Worried (3)

Not At All Worried (4)

I'm not sure (5)

Q10 Do you understand what is meant by contact tracing for an infectious disease like COVID-19?

Yes (1)

No (2)

I'm not sure (3)

Q11 Would you be happy to tell public health doctors where you have been in the past 2 weeks if it was important to stop the spread of COVID-19?

Yes (1)

No (2)

I'm not sure (3) 
Q12 Would you be happy to tell public health doctors who you have met in the past 2 weeks if it was important to stop the spread of COVID-19?

Yes (1)

No (2)

I'm not sure (3)

Q13 Do you own a mobile phone?

Yes (1)

No (2)

Q14 Is it a smartphone with a large glass screen OR does it have large buttons that you can press individually (i.e. an older design)?

Newer smartphone design (1)

Older more basic design (2)

I'm not sure (3) 
Q15 Which statement best describes where you generally keep your mobile phone during the day?

I keep it very close by (e.g. in pocket or bag or on desk) all the time (1)

I keep it close by (e.g. in pocket or bag or on desk) more than $50 \%$ of the time but not all the time (2)

I keep it close by (e.g. in pocket or bag or on desk) less than $50 \%$ of the time (3)

I rarely have my phone close by during the day (4)

I'm not sure (5)

Q16 Have you ever downloaded a new software Application (App) to your phone?

Yes (1)

No (2)

I'm not sure (3)

Q17 Would you need someone to show you how to download a new App to your phone?

Yes (1)

No (2)

I'm not sure (3) 
Q18 Has technology helped you overcome any barriers related to COVID-19 restrictions in Ireland? (please select any that apply)

Yes- Technology has helped me to work from home (1)

Yes- Technology has helped me to keep in touch with friends and family (2)

Yes- Technology has helped me to keep in touch with my local congregation/religious service (3)

Yes- Technology has helped me in some other way (please specify) (4)

QNo- While I have used technologies/Apps since COVID-19 restrictions began, they have not helped me overcome barriers (5)

$\bigotimes_{\text {No-I haven't used any technologies/Apps since COVID-19 restrictions began (6) }}$

\section{Q19 Mobile Phone Technology}

Mobile phones use a variety of wireless technologies to communicate with other phones, computers or networks.

These Mobile phone technologies can be used to send images nearby to a friend, control appliances in your home, connect to the internet, or accurately log your location using satellite technology (such as Global Positioning System (GPS)).

Please try and answer these questions even if you're not sure what these technologies are used for! Most mobile phones today come with "Bluetooth" technology installed. Do you understand what Bluetooth technology is used for?

Yes (1)

No (2)

I'm not sure (3) 
Q20 Does turning on Bluetooth on your mobile phone affect your battery life?

Yes (1)

No (2)

I'm not sure (3)

Q21 Do you know how to turn on Bluetooth on your mobile phone?

Yes (1)

No (2)

I'm not sure (3)

Q22 How often do you turn on Bluetooth on your mobile phone?

Everyday (1)

Most days (2)

Some days (3)

Rarely (4)

Never (12)

I'm not sure (13) 
Q23 What do you turn Bluetooth on for?

Q24 All phones can to some degree be used to work out your position. This can use GPS location, what phone mast you are near, what wireless network you are connected to etc.

Have you used your phone's "GPS location" or "Geolocation" technology before?

Yes (1)

No (2)

I'm not sure (3)

Q25 What have you used your GPS location/Geolocation for? 
Q26 HSE Contact Tracing App Currently in Ireland if someone tests positive for COVID-19, the HSE public health team contact them by telephone call to inform them and ask them who they have recently came into contact with.

But now let's imagine there is an Irish HSE App which you can download to your phone.

It does not access your files, photos or videos.

However, it does record when it comes into close contact with another phone which also has the App. This information is stored on your phone, and can only be released with your permission.

If 2 phones with the App installed come into close contact with each other, and 1 of their owners then develops COVID-19, this information could be used to help warn other people with the App who might be at higher risk of developing COVID-19.

If such an App is launched in the near future, how likely would you be to install the App on your smartphone?

Definitely will install (1)

Probably will install (2)

May or may not install (3)

Probably won't install (4)

Definitely won't install (5)

Not applicable (6)

Q27 What is the main reason, if any, you would install such an App? 
Q28 Please select any other reasons that would make it more likely for you to install the App (you can select many answers)

It would let me know my risk of being infected (1)

It would help protect me (2)

It would help protect my family and friends (3)

A sense of responsibility to the wider community (4)

It would help reduce the number of deaths among older people (5)

It might stop the epidemic (6)

Other (please specify) (7)

$\bigotimes$ None of these apply to me (8)

Q29 What is the main reason, if any, you would not install such an App? 
Q30 Please select any other reasons that would make it less likely for you to install the App (you can select many answers)

I've already had COVID-19 (1)

I won't be infected anyway (2)

The App would be too much hassle to install (3)

I would not benefit from the App (4)

I worry that my phone would be more likely to get hacked (5)

I worry the technology companies would use this as an excuse for greater surveillance after the epidemic (6)

I worry the government would use this as an excuse for greater surveillance after the epidemic (7)

I don't want to feel more anxious than I already feel (8)

I wouldn't give the HSE access to information about which phones or people I have been near recently (9)

I wouldn't give the HSE access to information about which locations I have visited recently (10)

Other (please specify) (11)

None of these apply to me (12) 
Q31 Does the technology that would be needed to allow an App like this to work matter to you (i.e. Bluetooth or Geolocation/GPS technology)?

You may wish to bear in mind that Bluetooth-based Apps do not need to access your geolocation/GPS data, whereas Geolocation-based Apps do.

I'd prefer a Bluetooth-only App (1)

I'd prefer a Geolocation/GPS-only App (2)

I'd prefer the App to use both Bluetooth and Geolocation technologies (3)

No, the technology used does not matter to me (4)

I'm not sure (5)

Q32 If you needed to turn on your Bluetooth for the App to work, would you do this?

Yes, I would turn on my phone's Bluetooth all the time (1)

Yes, I would turn on my phone's Bluetooth when leaving the house (2)

Yes, but only in the following circumstances... (please specify) (3)

No, it would be a hassle (4)

No, I don't know how (5)

No, for some other reason... (please specify) (6)

Not applicable (7)

I'm not sure (8) 
Q33 If the App needed to access to your GPS locations/Geolocation to work, would you allow it?

Yes, I would allow the App to use the Geolocation capabilities of my smartphone (1)

No, I wouldn't allow the App to use the Geolocation capabilities of my smartphone (2)

Not applicable (3)

I'm not sure (4)

Q34

If you tested positive for COVID-19 at some point in the future, would you be happy to agree that the HSE would be able to access your phone's record of potential close contacts?

Note, as with all questions, your answer is anonymous and will not commit you to any course of action when the App arrives

Yes (1)

No (2)

Not applicable (3)

I'm not sure (4) 
Q35

We hope these questions have helped you have a think about the potential risks and potential benefits of a contact tracing App and how the different technologies that would be needed work.

Please select again how likely you would be install the App on your smartphone...

Definitely will install (1)

Probably will install (2)

May or may not install (3)

Probably won't install (4)

Definitely won't install (5)

Not applicable (6)

Q36 Do you have any other comments on what you think of the proposed HSE contact tracing App?

Yes (please specify) (4)

No (5)

Q37 Last question, do you have any other feedback on this survey or research project?

Yes (please specify) (4)

No (5) 


\section{References}

1. World Health Organisation (WHO). Coronavirus disease 2019 (COVID-19) situation report-44 (March 4, 2020). Available at https://www.who.int/docs/default-source/coronaviruse/situationreports/20200304-sitrep-44-covid-19.pdf?sfvrsn=93937f92_6 (accessed March 5, 2020)

2. National Health Library and Knowledge Service Evidence Team, Health Service Executive (HSE), Ireland. Mar 2020. Rapid evidence review: Covid-19 contact tracing. Lenus, the Irish Health Repository Mar 2020. Available at: https://www.lenus.ie/handle/ 10147/627288 (accessed 07/06/20)

3. Health Information and Quality Authority (HIQA), Ireland. Evidence summary for asymptomatic transmission of COVID-19. Apr 2020. Available at: https://www.hiqa.ie/sites/default/files/ 2020-04/Evidence-summary-for-asymptomatic-transmission-ofCOVID-19.pdf (accessed 07/06/20)

4. Gandhi M, Yokoe DS, Havlir DV (2020) Asymptomatic transmission, the Achilles' heel of current strategies to control Covid-19. N Engl J Med. Editorial. Available at: https://www.nejm.org/doi/full/ 10.1056/NEJMe2009758 (accessed 07/06/20)

5. European Centre for Disease Prevention and Control (ECDC). Contact tracing for COVID-19: current evidence, options for scale-up and an assessment of resources needed. Apr 2020. Available https://www.ecdc.europa.eu/sites/default/files/ documents/COVID-19-Contract-tracing-scale-up.pdf (accessed 07/06/20)

6. Bengio Y, Janda R, Yu YW et al (2020) The need for privacy with public digital contact tracing during the COVID-19 pandemic. Lancet Digit Health 0(0) Available at https://www.thelancet.com/ journals/landig/article/PIIS2589-7500(20)30133-3/fulltext (accessed 07/06/20)

7. Nature- Editorial 580, 563 Apr 2020. Show evidence that apps for COVID-19 contact-tracing are secure and effective. Available at https://www.nature.com/articles/d41586-020-01264-1 (accessed 07/06/20)

8. Hinch R, Probert W, Nurtay A et al. Effective configurations of a digital contact tracing App: a report to NHSX Available at https:// 045.medsci.ox.ac.uk/files/files/report-effective-app-configurations. pdf (accessed 07/06/20)

9. Bay J, Kek J, Tan A et al BlueTrace: a privacy-preserving protocol for community-driven contact tracing across borders government technology agency, Singapore. Available at https://bluetrace.io/static/ bluetrace whitepaper-938063656596c104632def383eb33b3c.pdf (accessed 07/06/20)

10. World Health Organization (WHO). Contact tracing in the context of COVID-19: interim guidance, 10 May 2020. Available at https:// apps.who.int/iris/handle/10665/332049 (accessed 07/06/20)

11. European Commission website. Coronavirus: a common approach for safe and efficient mobile tracing apps across the EU European Commission - Questions and Answers. May 2020. Available at https://ec.europa.eu/commission/presscorner/detail/en/qanda_20_ 869 (accessed 07/06/20)

12. Google. Exposure Notifications: using technology to help public health authorities fight COVID-19. Available at https://www. google.com/covid19/exposurenotifications/ (accessed 07/06/20)

13. Apple. Privacy-preserving contact tracing. Available at https:// www.apple.com/covid19/contacttracing (accessed 07/06/20)

14. Department of Health (DOH), Ireland. National App for COVID19. Available at https://www.gov.ie/en/news/d2a00d-national-appfor-covid-19/ (accessed 07/06/20)

15. Peeri NC, Shrestha N, Rahman MS et al (2020) The SARS, MERS and novel coronavirus (COVID-19) epidemics, the newest and biggest global health threats: what lessons have we learned? Int J
Epidemiol. Available at https://academic.oup.com/ije/advancearticle/doi/10.1093/ije/dyaa033/5748175 (accessed 07/06/20)

16. Qualtrics Core XM Survey Software. Qualtrics (2020) Provo, Utah, USA. Available at https://www.qualtrics.com/uk/core-xm/surveysoftware/ (accessed 20/06/20)

17. Ward H, Atchison C. Public response to UK government recommendations on COVID-19: population survey, 17-18 March 2020 Patient Experience Research Centre (PERC) of Imperial College London, School of Public Health

18. Abeler J, Altmann S, Milsom L et al Support in the UK for appbased contact tracing of COVID-19. Report from 14th April 2020. Department of Economics, University of Oxford. Available at https://osf.io/3k57r/ (accessed 18/06/20)

19. R: A language and environment for statistical computing. $\mathrm{R}$ Foundation for Statistical Computing, Vienna, Austria. Available at https://www.R-project.org/ (accessed 18/06/20)

20. Central Statistics Office (CSO), Ireland. Census of Population 2016 - Profile 10 Education, Skills and the Irish Language Available at https://www.cso.ie/en/releasesandpublications/ep/p-cp10esil/ p10esil/le/ (accessed 10/06/20)

21. Healthy Ireland Summary report 2019 Department of health, Ireland. 2020. Available at https://assets.gov.ie/41141/ e5d6fea3a59a4720b081893e11fe299e.pdf (accessed 10/06/20)

22. Global Mobile Consumer Survey 2019: The Irish cut Deloitte Ireland 2019. Available at https:/www2.deloitte.com/ie/en/pages/ technology-media-and-telecommunications/articles/global-mobileconsumer-survey.html (accessed 10/06/20)

23. Wright KB (2005) Researching internet-based populations: advantages and disadvantages of online survey research, online questionnaire authoring software packages, and web survey services. J Comput Mediated Commun 10(3):JCMC1034 Available at https://academic.oup.com/jcmc/article/10/3/JCMC1034/4614509 (accessed 20/06/20)

24. Bracken CC, Jeffres LW, Neuendorf KA, Atkin D (2009) Parameter estimation validity and relationship robustness: a comparison of telephone and internet survey techniques. J Telematics Inform 26(2):144-155 Available at https://www.sciencedirect. com/science/article/pii/S0736585308000208 (accessed 20/06/20)

25. European Data Protection Board (2020) Statement on the processing of personal data in the context of the COVID-19 outbreak. Available at https://edpb.europa.eu/sites/edpb/files/files/news/ edpb_statement_2020_processingpersonaldataandcovid-19_en.pdf (accessed 10/06/20)

26. Dixon H. Fears grow over contact tracing app's security and privacy The Telegraph. $5^{\text {th }}$ May 2020. Available at https://www. telegraph.co.uk/news/2020/05/05/fears-grow-contact-tracing-appssecurity-privacy/ (accessed 17/9/20)

27. Farries E. Covid-tracing app may be ineffective and invasive of privacy The Irish Times. $5^{\text {th }}$ May 2020. Available at https://www. irishtimes.com/opinion/covid-tracing-app-may-be-ineffective-andinvasive-of-privacy-1.4244638 (accessed 17/9/20)

28. Stokel-Walker C Can mobile contact-tracing apps help lift lockdown? British Broadcasting Corporation. $16^{\text {th }}$ April 20. Available at https://www.bbc.com/future/article/20200415-covid-19-couldbluetooth-contact-tracing-end-lockdown-early (accessed 17/9/20)

29. Milsom L, Abeler J, Altmann S et al (2020) Survey of acceptability of app-based contact tracing in the UK, US, France, Germany and Italy, Open Science Foundation (OSF) Website. Available at https://osf.io/7vgq9/wiki/home/ (accessed 07/06/20)

30. Trace Together Website. Ministry of Health, Singapore. Available at https://www.tracetogether.gov.sg/ (accessed 07/06/20)

31. A Representative Sample of Australian Participants's attitudes towards government tracing during the COVID-19 Pandemic. Dennis S, Garrett P, White J, Little D, Perfors A, Kashima Y, Lewandowsky S. Complex Human Data Hub, School of Psychological Sciences, The University of Melbourne. Available 
at https://paulgarrettphd.github.io/Site/Wave2Final.html (accessed 07/06/20)

32. A Representative Sample of Australian Participant's Attitudes Towards the COVIDSafe App. Garrett P, White J, Little D, Perfors A, Kashima Y, Lewandowsky S, Dennis S. Complex Human Data Hub, School of Psychological Sciences, The University of Melbourne. Available at https://paulgarrettphd. github.io/Site/Wave3PrelimAnalysis.html (accessed 07/06/20)

33. Meixner S. How many people have downloaded the COVIDSafe app and how central has it been to Australia's coronavirus response? ABC News Australia. Available at https://www.abc.net. au/news/2020-06-02/coronavirus-covid19-covidsafe-app-howmany-downloads-greg-hunt/12295130 (accessed 10/06/20)

34. The Rakning C-19 tracing app website. The Directorate of Health, Iceland. Available at https://www.covid.is/app/en (accessed 10/06/ 20)

35. Findlay S, Palma S, Milne R (2020) Coronavirus contact-tracing apps struggle to make an impact The Financial Times online edition. Available at https://www.ft.com/content/21e438a6-32f243b9-b843-61b819a427aa (accessed 10/06/20)

36. Phartiyal S. India follows China's lead to widen use of coronavirus tracing app Reuters News Agency. 14th May 2020. Available at https://www.reuters.com/article/health-coronavirus-india-app/ india-follows-chinas-lead-to-widen-use-of-coronavirus-tracingapp-idUSL4N2CV4JL (accessed 18/06/20)

37. Principles for legislators on the implementation of new technologies. Irish Council for Civil Liberties (ICCL). June 2020. Available at https://www.iccl.ie/wp-content/uploads/2020/06/Principles-forlegislators-on-the-implementation-of-new-technologies.pdf (accessed 10/06/20)

Publisher's note Springer Nature remains neutral with regard to jurisdictional claims in published maps and institutional affiliations. 